\title{
Clinical Leadership and Asymmetric Information: The Impact of Physician Advice on Smoking Cessation
}

\author{
Courtney Keeler \\ School of Nursing and Health Professions, University of San Francisco, San Francisco, USA \\ Email: ckeeler@usfca.edu
}

Received 8 April 2014; revised 8 May 2014; accepted 16 May 2014

Copyright (C) 2014 by author and Scientific Research Publishing Inc. This work is licensed under the Creative Commons Attribution International License (CC BY). http://creativecommons.org/licenses/by/4.0/

(c) (i) Open Access

\begin{abstract}
A knowledge gradient exists between experts in a given field and consumers of that knowledge. When the need arises, not knowing the best path, an average individual typically relies on the advice of an expert. Given the steep knowledge gradient between patient and provider, clinicians play an essential role in the clinical setting, acting as both a health leader and a health facilitator; however, this asymmetric information implies that clinical providers face an acute pressure not only to advise but to advise correctly. This paper explores the importance of physician advice within the context of smoking cessation, addressing two specific research questions: (1) among current smokers, do patients have a higher probability of any quit attempts in the last twelve months if a physician advised them to quit over the same period? and, (2) among current smokers who were advised to quit, do patients have a higher probability of any quit attempts in the past twelve months based, at least in part, on the specific quitting strategy suggested by the physician? The results suggest that physicians play a crucial role in promoting smoking cessation efforts. The findings further highlight a significant association between the advised cessation strategy and any quit attempts, although the direction of this relationship varies by the cessation strategy suggested.
\end{abstract}

\section{Keywords}

Healthcare Leadership, Health Economics, Substance Use, Smoking Cessation

\section{Introduction}

Rodney Dangerfield once joked, “I met the surgeon general—he offered me a cigarette.” Like many good jokes, 
the comedian touches on a fundamental truth: for better or worse, the advice as well as implicit or explicit endorsements by leaders in a field matter. A person cannot be a universal expert. Barring superhuman intellect, most individuals will not be well versed in everything from Shakespeare to hadron colliders. Therefore, a knowledge gradient exists between experts in a given field and consumers of that knowledge. When the need arises, not knowing the best path, an average individual typically relies on the advice of an expert.

In the context of healthcare, this knowledge gradient is particularly steep. Healthcare is characterized by a large degree of asymmetric information. As Phelps (2012) describes, the asymmetry exists since "one party (the doctor) generally has considerably, possibly massively, greater level of knowledge than the other (the patient) about the issues at hand, namely the diagnosis and treatment of disease,” (pg. 5). Without the clinician’s training and experience, patients cannot fully understand diagnostics and treatment strategies or preform essential medical procedures. As such, clinicians serve as essential and de facto leaders in the healthcare field, serving a constituency of patients who rely on their advice and medical services.

This asymmetrical information reinforces the clinician's role as a gatekeeper, shaping a patient's treatment trajectory. For example, despite the large degree of direct-to-consumer advertising, a patient cannot simply prescribe herself medication. Patients legally gain access only when their clinician writes a prescription. Resultantly, clinicians not only represent leaders within the health care field but also facilitators of patient health, reinforcing the theory that, in field of healthcare, health leadership requires facilitation (Keeler, 2013).

Given the dramatic knowledge gradient, clinical providers face an acute pressure to not only advise but to advise correctly. Rathert and colleagues (2011) supports this premise. Drawing on qualitative patient data, the authors explore acute care patients' perceptions of their role in promoting patient safety. Rathert and colleagues (2011) conclude, "[o]verall, most patients appeared to be suggesting that patients should listen, trust, and be cooperative," (pg. 139). In fact, one respondent described a patient's responsibilities as follows, "[t]o listen carefully and follow directions of your care givers,” (pg. 138). The work of Rathert and colleagues (2011) indicates that many patients take a backseat role in their care, relying on the wisdom of their caregivers. Given that there are often multiple solutions to a single problem, providers face the perilous task of advising the most efficacious route.

This paper explores the importance of clinicians' advice within the context of smoking cessation. Drawing on data from the 2010-2011 Tobacco Use Supplement to the Current Population Survey (TUS-CPS), this paper addresses two specific questions: (1) among current smokers, do patients have a higher probability of any quit attempts in the last twelve months if a physician advised them to quit over the same period? and, (2) among current smokers who were advised to quit, do patients have a higher probability of any quit attempts in the past twelve months based, at least in part, on the specific quitting strategy suggested by the physician? While multiple cessation aids exist, this paper focuses on five categories: telephone quit lines, smoking cessation classes, programs, or counseling, nicotine products like a patch or gum, prescription products such as Chantix, and setting a quit date.

This paper is organized as follows: Section two provides a brief background on smoking and smoking cessation in the United States; section three outlines the methods used, including the data source, sample population, key measures, and statistical analysis; section four details the empirical results; and, the fifth and final section discusses the implications of these findings in the context of clinical leadership.

\section{Background}

Launched in December of 2010 by the department of Health and Human Services, Healthy People 2020 sets a series of health-related objectives to be achieved by 2020, with the vision of creating "a society in which all people live long, healthy lives,” (US HHS, 2012). In particular, Healthy People outlines three broad goals surrounding tobacco use; the first of which includes the "[implementation of] policies to reduce tobacco use and initiation among youth and adults,” (CDC, 2013). Thus, the department of Health and Human Services explicitly identifies tobacco use as a crucial barrier obstructing the creation of a healthy, long-living society.

In 1964, the surgeon general released a seminal report, which, for the first time, linked smoking with a number of adverse outcomes, including all-cause mortality, cancer, and chronic bronchitis (US HHS, 2014). Since then, researchers have related smoking with a variety of poor health outcomes, including some unlikely health conditions such as diabetes mellitus, rheumatoid arthritis, and colorectal cancer (US HHS, 2014). Facing an ever increasing body of evidence demonstrating the perils associated with smoking, local, state, and federal governments continue to institute policy and regulation aimed at stemming smoking and other tobacco behaviors, in- 
cluding the recent federal Family Smoking Prevention and Tobacco Control Act passed in June 2009 (Pub.L 111-31, H.R. 1256).

Aimed at curbing smoking initiation by adolescents and young adults, the Food and Drug Administration (FDA, 2013) describes the Tobacco Control Actas "[restricting] tobacco product advertising and marketing to youth by directing [the] FDA to issue regulations," including the prohibition of "reduced harm" claims, further restrictions on packaging and advertisements, the elimination of free cigarette samples, and the proscription of tobacco sponsorship of sporting and entertainment events (FDA, 2013). As a result of the act, "Cigarette package health warnings will be required to cover the top 50 percent of both the front and rear panels of the package, and the nine specific warning messages must be equally and randomly displayed and distributed in all areas of the US," (FDA, 2013) ${ }^{1}$.

Despite public health awareness campaigns, legislation and regulation, the US currently faces a tobacco use epidemic-approximately eighteen percent of US adults age eighteen and over smoke cigarettes (CDC, 2014). Indeed, smoking remains the leading preventable cause of premature death in the US (US HHS, 2014).

Not surprisingly, the economic cost of smoking in terms of health care resources is huge, ranging between $0.6 \%$ and $0.85 \%$ of US gross domestic product (Parrot and Godfrey, 2004). As such, further facilitation of cessation efforts present obvious benefits, both in terms of health as well as financial savings.

In terms of health benefits, at an individual level, cessation is associated with an increase in life expectancy and a decrease in the incidence of comorbid disease; self-reported health status also increases, suggesting an improvement in an individual's overall quality of life (Parrot and Godfrey, 2004). Individuals residing with former smokers share in this improvement, benefiting from reduced exposure to secondhand smoke and the associated complications (Parrot and Godfrey, 2004).

Given the economic and health-related benefits, naturally, an expansive body of research explores the incidence of smoking cessation; nevertheless, experts agree that smoking cessation merits more attention (HHS, 2014). Most literature defines the incidence of successful cessation as the ratio of newly successful former smokers in a given year divided by recent active smokers who are eligible to quit (e.g., Gilpin and Pierce, 2002; Chang et al., 2014). "Success" is gauged by having quit for a specific and sufficient period of time, which, in the literature, usually ranges from three to six months. For instance, Chang and colleagues (2014) define the annual cessation rate as quitting for at least three months among recent active smokers.

In the United States, the prevalence of cigarette cessation has increased over time. Using National Health Interview Survey data, Gilpin and Pierce (2002) find that, among US adults, the "incidence [of quitting] increased 5 fold” between 1962 and 1992. Exploring smoking behaviors over the subsequent decade (1992 to 2002), Pierce and colleagues (2009) observe a continued reduction in the prevalence of cigarette consumption. Drawing on the 1992-1993, 1998-1999, and 2006-2007 Tobacco Use Supplement to the Current Population Survey, Jemal and colleagues (2011) observe a decrease in the prevalence of smoking between 1992 and 2007.

Public awareness campaigns, legislation, and regulation shape cessation patterns. For example, Gilpin and Pierce (2002) note that following the surgeon general's report detailing the adverse effects of smoking on health, including the health of fetuses, the incidence of cessation increased among young, female smokers in the 1970s. The authors also note the positive effect of 1980's public awareness campaigns aimed at curbing smoking. Jemal and colleagues (2011) find a strong, negative correlation between the decreased prevalence of smoking and state-level policies regarding cigarette excise taxes and indoor air.

In some cases, the influence of public awareness campaigns, legislation, and regulation is paralleled in other countries. For instance, investigating the impact of the multi-pronged 2009 Taiwanese Tobacco Hazards Prevention Act, which "[extended] smoke-free areas to include almost all enclosed work-places and public places, [added] graphic health warnings to cigarette packages, totally [banned] tobacco advertisements, promotion and sponsorship and [increased] tobacco taxes,” (pg. 140), Chang and colleagues (2014) find that, taken together, the legislation was associated with a statistically significant increase in quit attempts as well as the annual cessation rate.

Importantly, demographic and other factors mediate cessation trends. Some dramatic examples surround race/ethnicity, socioeconomic indicators, and price. For instance, African Americans consistently exhibit lower quit rates relative to Caucasians (e.g., Gilpin and Pierce, 2002), although the impact of education seems to su-

\footnotetext{
${ }^{1}$ While a step in the right direction, the Tobacco Control Act inevitably contains loopholes. For instance, while banning flavoring in cigarettes, menthol flavoring is still permissible. This caveat is of particular importance in the context of smoking cessation since, as Trinidadand colleagues (2010) note, "those who used to regularly smoke mentholated cigarettes were less likely to have experienced long-term quitting success,” (pg. 84).
} 
persede that of race/ethnicity (Gilpin and Pierce, 2002; Keeler et al., 1999). Socioeconomic factors appear to further mediate the impact of price. Indeed, some research suggests that individuals of lower socioeconomic status are more responsive to price (e.g., Townsend et al., 1994). Investigating the prevalence of smoking in Caucasian and African American populations in the southeast and acknowledging the strong correlation between socioeconomic indicators and smoking outcomes, Cohen and colleagues (2011) emphasize the importance of targeted interventions in high-risk populations. Gender also plays a role; for instance, Townsend and colleagues (1994) find that, relative to men, women are less responsive to public health campaigns and more responsive to changes in price.

Building on Cohen and colleagues (2011) argument for targeted interventions, no intervention can be more targeted than the cessation strategy devised between a patient and her clinical provider. As such, one of the first frontiers of the battle for smoking cessation takes place in the clinical setting. Acting not only as a health leader but health facilitator, the clinical provider plays an important role in promoting cessation behaviors. A clinician can help initiate cessation efforts by simply broaching the idea of quitting during a patient's visit; furthermore, as suggested by the aforementioned literature, the manner in which a clinician advises cessation and the strategies that he or she recommends likely influences the patient's probability of success.

\section{Methods}

\subsection{Data Source}

The data for this analysis come from the 2010-2011 Tobacco Use Supplement to the Current Population Survey (TUS-CPS). Backed by the US Bureau of Labor Statistics and US Bureau of Census, the Current Population Survey (CPS) is a nationally representative, primarily cross-sectional survey. The CPS serves as the chief source of nationally representative economic and labor market data, administered monthly to approximately 60,000 households. In addition to these employment data, the CPS also collects essential demographic information.

The CPS is often accompanied by supplemental surveys. In particular, the National Cancer Institute periodically sponsors a tobacco use supplement (TUS), which collects data on, among other things, current smoking status, tobacco use history, smoking behaviors, tobacco products used, and attitudes towards smoking. Of importance for the present analysis, the 2010-2011 TUS data contain information on cessation recommendations by a medical doctor.

\subsection{Sample Population}

The 2010-2011 TUS questionnaire was administered in May 2010, August 2010, and January 2011; observations across these three waves are consolidated to create a single, pooled cross-section. This study focuses on the quit attempts of current, active smokers. Current, active smokers are defined as individuals who identify as everyday smokers or someday smokers who smoked twelve days or more in the past thirty ${ }^{2}$. As such, former smokers and individuals who have never smoked are excluded from the sample ${ }^{3}$. The sample population is further restricted to individuals age eighteen to sixty-four to enhance the external validity of the results within the context of an adult, working-age population. The original pooled cross-section contained 18,345 self-respondents who identified as current, active smokers age eighteen to sixty-four, who smoke every day or at least twelve days in the last thirty. Of this group, 5,139 respondents indicated that they had not visited a doctor in the last twelve months and were, therefore, excluded from the sample. Observations with incomplete information for the relevant variables were also excluded from the analysis, resulting in a final sample size of 11,726 individuals.

\subsection{Outcome Measures}

Similar to Chang and colleagues (2014), this paper explores the prevalence of having any quit attempts in the last twelve months. The TUS questionnaire asks, "During the past 12 months, have you stopped smoking for one day or longer because you were trying to quit?” Therefore, the denominator of this prevalence measure includes

\footnotetext{
${ }^{2}$ The outcome measure is only asked of someday smokers who smoked twelve or more days in the past thirty, thus limiting the sample population to someday smokers who meet this threshold.

${ }^{3}$ Cessation studies often encompass "recent, active smokers," including former smokers who quit in the last twelve months in the sample population; nevertheless, questions relating to physician advice are only asked of someday and everyday smokers, limiting the sample to current smokers.
} 
all current daily smokers and someday smokers who smoked twelve or more days in the past thirty; the numerator reflects the number within this group who have quit one day or longer with the intention to quit smoking.

\subsection{Physician Measures}

Among those individuals who saw a medical doctor in the past twelve months, the TUS questionnaire asks someday and everyday smokers whether any medical doctor advised them to quit smoking. Based on this question, a dummy variable was created to reflect whether a medical doctor advised the patient to quit (variable referred to as ADVISE).

Among those individuals who were advised to quit smoking by a medical doctor, the TUS questionnaire asks whether "In the PAST 12 MONTHS, when a medical doctor advised you to quit smoking, did the doctor also":

- "Suggest that you call or use a telephone help line or quit line"

- "Suggest that you use a smoking cessation class, program, or counseling?”

- “Recommend or prescribe a nicotine product such as a patch, gum, lozenge, nasal spray or inhaler?”

- "Prescribe a pill such as Chantix, Varenicline, Zyban, Bupropion, or Wellbutrin?

- "Suggest that you set a specific date to stop smoking?”

These five categories are transformed into five separate binary indicators, reflecting whether a physician advised the use of a help line, class/program/counseling, a nicotine product, other prescription pill, and setting a quit date (variables referred to as HELP, CLASS, NICOTINE, PILL, and DATE, respectively).

\subsection{Demographic Measures}

In addition to the physician indictors, demographic and socioeconomic variables are incorporated into the analysis. These variables include indicators relating to gender, race/ethnicity, family income, education, marital status, employment status, and geographic region (Northeast, West, South, and Midwest). These demographic variables capture the key mediating factors discussed in the previous background section.

\subsection{Statistical Analysis}

The data are analyzed using Stata 13, a statistical software package. All estimates are weighted. Mirroring Chang and colleagues' (2014) statistical analysis, the paper first presents the results from a bivariate chi-squared test, which assesses differences in quit attempts across physician indicators and other demographic categories.

Next, the paper uses logistic regression techniques to investigate whether physician cessation advice over a twelve-month period is positively associated with quitting for at least one day over the same period. Specifically, the paper presents the results from two multivariate logistic regressions. The first analysis regresses any quit attempts on the physician advice variable (ADVISE) and the remaining covariates. Among those individuals whose physicians advised them to quit, the second analysis regresses any quit attempts on the five cessationstrategy indicators (HELP, CLASS, NICOTINE, PILL, and DATE) and the remaining covariates.

\section{Results}

\subsection{Demographic Characteristics}

Table 1 presents basic summary statistics. The sample population consists of current, active smokers age eighteen to sixty-four, who smoke every day or at least twelve days in the last thirty. The sample population is approximately equally divided between men and women. The population is also equally distributed between the three narrow age categories (eighteen to twenty-four; twenty-five to forty-nine; and, fifty to sixty-four). The typical or average person in the sample identifies as Caucasian, has a family income between $\$ 50,000$ and $\$ 74,999$ per year, has a high school education, is married, and employed. Sixty-six percent of the population has been advised by a medical doctor to quit smoking.

\subsection{Prevalence of Any Quit Attempts in the Past Twelve Months among Current Smokers by Demographic Characteristics}

Table 2 presents prevalence of any quit attempts in the past twelve months (i.e., quit for at least one day with the intention of quitting). A bivariate chi-squared test assesses differences in quit attempts across physician in 
Table 1. Descriptive statistics.

\begin{tabular}{|c|c|c|c|}
\hline & $\mathrm{N}$ & Mean & SE \\
\hline Advise on smoking cessation in last 12 months & 11,726 & 1.00 & \\
\hline Doctor did not advise to quit & & 0.339 & $(0.00495)$ \\
\hline Doctor advised to quit & & 0.661 & $(0.00495)$ \\
\hline \multicolumn{4}{|l|}{ Cessation strategy advised by doctor in last 12 months } \\
\hline Quit line & 8222 & 1.000 & \\
\hline Quit line not suggested & & 0.801 & $(0.00495)$ \\
\hline Quit line suggested & & 0.199 & $(0.00495)$ \\
\hline Class/program/counseling & 8222 & 1.000 & \\
\hline Class/program/counseling not suggested & & 0.827 & $(0.00468)$ \\
\hline Class/program/counseling suggested & & 0.173 & $(0.00468)$ \\
\hline Nicotine product & 8222 & 1.000 & \\
\hline Nicotine product not suggested & & 0.730 & $(0.00549)$ \\
\hline Nicotine product suggested & & 0.270 & $(0.00549)$ \\
\hline Prescription product & 8222 & 1.000 & \\
\hline Prescription product not suggested & & 0.756 & $(0.00529)$ \\
\hline Prescription product suggested & & 0.244 & $(0.00529)$ \\
\hline Setting a quit date & 8222 & 1.000 & \\
\hline Quit date not suggested & & 0.807 & $(0.00491)$ \\
\hline Quit date suggested & & 0.193 & $(0.00491)$ \\
\hline Gender & 11,726 & 1.000 & \\
\hline Female & & 0.512 & $(0.00878)$ \\
\hline Male & & 0.488 & $(0.00878)$ \\
\hline Age & 11,726 & 1.000 & \\
\hline 18 to 24 (Omitted) & & 0.334 & $(0.00531)$ \\
\hline 25 to 49 & & 0.324 & $(0.00477)$ \\
\hline 50 to 64 & & 0.342 & $(0.00482)$ \\
\hline Race/ethnicity & 11,726 & 1.000 & \\
\hline White (Omitted) & & 0.776 & $(0.00472)$ \\
\hline African American & & 0.109 & $(0.00355)$ \\
\hline Native American & & 0.00972 & $(0.000906)$ \\
\hline Asian & & 0.0174 & $(0.00147)$ \\
\hline Pacific islander & & 0.00165 & $(0.000396)$ \\
\hline Hispanic & & 0.0694 & $(0.00292)$ \\
\hline Other & & 0.0178 & $(0.00141)$ \\
\hline
\end{tabular}




\section{Continued}

\section{Family income}

$\$ 0$ to $\$ 24,999$

$\$ 25,000$ to $\$ 49,999$

$\$ 50,000$ to $\$ 74,999$

$\$ 75,000$ to $\$ 99,999$

$\$ 100,000+$ (Omitted)

Highest level of educational attainment

$>12$ th

12th or equivalent

Associate degree

Bachelor's degree

At least some graduate school (Omitted)

Marital status

Never married (Omitted)

Married

Widowed

Divorced

Separated

Employment status

Unemployed (Omitted)

Employed

Not in labor force

Geographic region

West (Omitted)

Northeast

Midwest

South
11,726

1.000

0.228

(0.00466)

0.206

(0.00455)

0.115

(0.00349)

0.0638

(0.00279)

0.388

(0.00511)

11,726

1.000

0.145

(0.00370)

0.631

(0.00493)

0.0999

(0.00296)

0.0978

(0.00307)

0.0270

(0.00157)

11,726

1.000

0.295

(0.00507)

0.430

(0.00532)

0.0314

(0.00167)

0.196

(0.00397)

0.0468

(0.00213)

11,726

1.000

0.108

(0.00335)

0.605

(0.00514)

0.287

(0.00469)

11,726

1.000

0.165

(0.00418)

0.174

(0.00452)

0.275

(0.00516)

0.385

(0.00572)

Notes: (a) All estimates weighted. (b) Sample population consists of all current everyday and some day smokers who smoked on at least 12 days in the last 30. (c) Cessation strategies (i.e., quit line, class/program/counseling, nicotine product, prescription product, and setting a quit date) only analyzed among those individuals who were advised to quit smoking by a medical doctor, resulting in a smaller sample size.

dicators and other demographic categories (as seen by the $p$-values in the table). The test indicates several key differences, including difference between those who were advised to quit by a medical doctor and those who were not. Among those individuals advised to quit by a medical doctor, the bivariate chi-squared test signals essential differences between those who were advised to use a class, program, or counseling compared to those who were not, those who were advised to use a prescription product compared to those who were not, and those who were advised to set a quit date compared to those who were not.

\subsection{Multiple Logistic Regression Analysis: The Relationship between Physician Advice and Any Quit Attempts}

Regression (1) in Table 3 details the results. As expected, the coefficient on physician advice is statistically sig- 
Table 2. Prevalence of any quit attempts in the past twelve months among current smokers by demographic characteristics.

\begin{tabular}{|c|c|c|c|c|}
\hline & $\%$ & \multicolumn{2}{|c|}{ 95\% CI (95 min, 95 max) } & $\begin{array}{r}p \text {-Value } \\
0.0000\end{array}$ \\
\hline Doctor did not advise to quit (Omitted) & $37.53 \%$ & $35.82 \%$ & $39.25 \%$ & \\
\hline Doctor advised to quit & $42.79 \%$ & $41.57 \%$ & $44.01 \%$ & \\
\hline \multicolumn{5}{|l|}{ Cessation strategy advised by doctor in last 12 months } \\
\hline Quit line & & & & 0.5531 \\
\hline Quit line not suggested (Omitted) & $42.97 \%$ & $41.60 \%$ & $44.34 \%$ & \\
\hline Quit line suggested & $42.07 \%$ & $39.43 \%$ & $44.71 \%$ & \\
\hline Class/program/counseling & & & & 0.0702 \\
\hline Class/program/counseling not suggested (Omitted) & $43.29 \%$ & $41.94 \%$ & $44.64 \%$ & \\
\hline Class/program/counseling suggested & $40.40 \%$ & $37.59 \%$ & $43.21 \%$ & \\
\hline Nicotine product & & & & 0.2494 \\
\hline Nicotine product not suggested (Omitted) & $43.21 \%$ & $41.78 \%$ & $44.65 \%$ & \\
\hline Nicotine product suggested & $41.65 \%$ & $39.38 \%$ & $43.92 \%$ & \\
\hline Prescription product & & & & 0.0000 \\
\hline Prescription product not suggested (Omitted) & $40.07 \%$ & $38.67 \%$ & $41.46 \%$ & \\
\hline Prescription product suggested & $51.24 \%$ & $48.86 \%$ & $53.62 \%$ & \\
\hline Setting a quit date & & & & 0.0000 \\
\hline Quit date not suggested (Omitted) & $40.42 \%$ & $39.06 \%$ & $41.77 \%$ & \\
\hline Quit date suggested & $52.74 \%$ & $50.03 \%$ & $55.45 \%$ & \\
\hline Gender & & & & 0.6483 \\
\hline Female (Omitted) & $41.23 \%$ & $39.93 \%$ & $42.52 \%$ & \\
\hline Male & $40.77 \%$ & $39.27 \%$ & $42.27 \%$ & \\
\hline Age & & & & 0.0000 \\
\hline 18 to 24 (Omitted) & $46.08 \%$ & $44.16 \%$ & $48.01 \%$ & \\
\hline 25 to 49 & $39.77 \%$ & $38.12 \%$ & $41.42 \%$ & \\
\hline 50 to 64 & $37.23 \%$ & $35.68 \%$ & $38.78 \%$ & \\
\hline Race/ethnicity & & & & 0.0007 \\
\hline White (Omitted) & $40.20 \%$ & $39.09 \%$ & $41.31 \%$ & \\
\hline African American & $46.24 \%$ & $42.99 \%$ & $49.48 \%$ & \\
\hline Native American & $42.45 \%$ & $33.33 \%$ & $51.56 \%$ & \\
\hline Asian & $34.60 \%$ & $26.69 \%$ & $42.51 \%$ & \\
\hline Pacific islander & $70.58 \%$ & $49.33 \%$ & $91.84 \%$ & \\
\hline Hispanic & $41.45 \%$ & $37.41 \%$ & $45.50 \%$ & \\
\hline Other & $45.45 \%$ & $37.83 \%$ & $53.07 \%$ & \\
\hline
\end{tabular}




\section{Continued}

\section{Family income}

$\$ 0$ to $\$ 24,999$

$\$ 25,000$ to $\$ 49,999$

$\$ 50,000$ to $\$ 74,999$

$\$ 75,000$ to $\$ 99,999$

$\$ 100,000+$ (Omitted)

Highest level of educational attainment

$>12$ th

12th or equivalent

Associate degree

Bachelor's degree

At least some graduate school (Omitted)

Marital status

Never married (Omitted)

Married

Widowed

Divorced

Separated

Employment status

Unemployed (Omitted)

Employed

Not in labor force

Geographic region

West (Omitted)

Northeast

Midwest

South
$40.70 \%$

$40.13 \%$

$38.78 \%$

$38.73 \%$

$42.69 \%$

$38.32 \%$

$41.00 \%$

$44.01 \%$

$41.55 \%$

$42.49 \%$

$44.01 \%$

$40.31 \%$

$36.23 \%$

$39.07 \%$

$39.88 \%$

$46.07 \%$

$40.96 \%$

$39.23 \%$

$42.21 \%$

$41.69 \%$

$42.42 \%$

$39.19 \%$
0.0824

$38.57 \% \quad 42.83 \%$

$37.91 \% \quad 42.35 \%$

$35.87 \% \quad 41.69 \%$

$34.66 \% \quad 42.80 \%$

$41.13 \% \quad 44.26 \%$

0.0813

$35.68 \% \quad 40.96 \%$

$39.74 \% \quad 42.27 \%$

$40.99 \% \quad 47.04 \%$

$38.40 \% \quad 44.70 \%$

$36.79 \% \quad 48.19 \%$

0.0013

$41.99 \% \quad 46.03 \%$

$38.85 \% \quad 41.78 \%$

$31.15 \% \quad 41.32 \%$

$37.01 \% \quad 41.13 \%$

$35.42 \% \quad 44.34 \%$

0.0009

$42.87 \% \quad 49.28 \%$

$39.68 \% \quad 42.24 \%$

$37.43 \% \quad 41.03 \%$

0.0407

$39.78 \% \quad 44.64 \%$

$39.23 \% \quad 44.14 \%$

$40.56 \% \quad 44.28 \%$

$37.56 \% \quad 40.81 \%$

Notes: (a) All estimates weighted. (b) Sample population consists of all current everyday and some day smokers who smoked on at least 12 days in the last 30. (c) The denominator of this prevalence measure includes all current daily smokers and someday smokers who smoked twelve or more days in the past thirty; the numerator reflects the number within this group who have quit one day or longer. (d) Sample sizes correspond to values detailed in Table 1. (e) $P$-values from chi-squared test.

nificant and positively associated with the probability of having quit at least for one day in the last twelve months. Among current smokers who had a medical doctor advise them to quit, the odds of quitting for at least one day with the intention to quit (versus not quitting for at least one day) increase by a factor of 1.315 relative to those smokers who did not receive such advice, adjusting for the additional covariates.

Additionally, younger smokers appear more likely to quit relative to older smokers. While the majority of race/ethnicity variables are insignificant, African Americans and Pacific Islanders appear more likely to quit smoking for one day or longer relative to Caucasian smokers ( $p<0.001$ and $p<0.05$, respectively). This result has some support in the literature. For instance, Keeler and colleagues (1999) find that African Americans have fewer failed quit attempts but also fewer successful occurrences of cessation. The odds of any quit attempts increase with education, supporting the existing literature (e.g., Gilpin and Pierce, 2002; Keeler et al., 1999). Compared to the unemployed, employed individuals and those individuals not part of the labor force have lower 
odd of quitting. This result may reflect one's finances-if cigarettes are a normal good (meaning, demand increases as income increases), those without income may quit simply because they cannot afford the expense. Finally, southerners have lower odds of quitting for at least one day with the intention quit relative to those living in the west $(p<0.05)$.

\subsection{Multiple Logistic Regression Analysis: The Relationship between Cessation Strategy Advice and Any Quit Attempts among Those Individuals Advised to Quit Smoking by a Medical Doctor}

Regression (2) in Table 3 details the results. The relationship between cessation strategy and the likelihood of quitting varies by the cessation strategy advised. With the exception of help/quit lines, all cessation strategies are statistically significant. The Wald test indicates that the cessation variables (HELP, CLASS, NICOTINE, PILL, and $D A T E)$ are jointly significant $(p<0.000)$. Nevertheless, the direction of these effects is not consistently positive.

Among those whose medical doctor suggested using or prescribed a product such as Chantix, the odds of quitting for at least one day with the intention to quit (versus not quitting for at least one day) increase by a factor of 1.564 relative to those smokers who did not receive such advice, adjusting for the additional covariates. Similarly, among current smokers whose doctor medical doctor suggested setting a quit date, the odds of quitting for at least one day with the intention to quit increase by a factor of 1.626 relative to those smokers who did not receive such advice, adjusting for the additional covariates. Interestingly, while both positive, the impact of setting a quit date seems to slightly supersede that of prescription drugs, at least in terms of the magnitude of the effect.

While having a medical doctor suggest prescription medications or setting a quit date in the last twelve months are positively associated with the odds of having quit for at least one day over the same period (versus those who did not receive this advice), other cessation recommendations relating to nicotine products and classes/counseling run in the opposite direction. Smokers advised to use a nicotine product had lower odds of any quit attempts in the last year relative to smokers who did not have a physician make such a recommendation. Similarly, smokers who were advised to attend a class, program, or counseling also had lower odds of quitting for at least one day compared to smokers who did not receive this advice.

Once again, several of the remaining covariates are significant, and run in the same direction as observed in regression (1).

\section{Discussion}

The results suggest that physicians play a crucial role in promoting smoking cessation efforts. The empirical analysis reveals that current smokers who were advised to quit by a medical doctor in the past twelve months had higher odds of quitting at least one day with the intention to quit over the same period compared to smokers who did not receive such advice.

Among those advised to quit by a medical doctor, the findings further highlight a significant association between suggested cessation strategies and any quit attempts in the last year, although the direction of this relationship varies by the cessation strategy recommended. Smokers advised to use a nicotine product or attend a class, program, or counseling had lower odds of quitting for at least one day relative to patients not recommended these strategies. Comparatively, smokers advised to use a prescription product like Chantix or set a quit date experienced a higher odds of any quit attempts relative to those who did not receive this advice.

These results complement the existing literature. For instance, summarizing their results, Fiore and colleagues (1990) write, "The cessation method used was the strongest predictor of success. Among smokers who had attempted cessation within the previous 10 years, $47.5 \%$ of persons who tried to quit on their own were successful whereas only $23.6 \%$ of persons who used cessation programs succeeded,” (pg. 2760). These results parallel the findings of the current work, which indicate that autonomously driven endeavors, like setting a quit date, are more strongly and positively associated with any quit attempts compared to classes, programs, or counseling.

Selection effects may partially explain the observed variation in the odds of any quit attempts across the varying cessation strategies. Cessation strategies may be associated with differing financial and time costs, drawing systematically different types of smokers. Indeed, we have already seen that income and smoking are related. Additionally, cessation strategies may also vary with smoking intensity. Perhaps a physician may be more likely to advise a nicotine product or class for heavy smokers; this may explain why smokers advised with these strategies are less likely to have any quit attempts. Finally, some cessation strategies may be gradual in nature, resulting in 
Table 3. Multiple logistic regressions assessing the association between physician advice and any quit attempts in the later twelve months.

Any quit attempts, past 12 months
Ant

Advise on smoking cessation in last 12 months

Doctor did not advise to quit

Doctor advised to quit

Adj. OR

CI

Reference

$1.315^{* * *}$

[1.203, 1.438]
(2)

Any quit attempts, past 12 months (Conditional on a medical doctor advising individual to quit)

CI

\section{Cessation strategy advised by doctor in last 12 months}

Quit line not suggested

Quit line suggested

Class/program/counseling not suggested

Class/program/counseling suggested

Nicotine product not suggested

Nicotine product suggested

Prescription product not suggested

Prescription product suggested

Quit date not suggested

Quit date

Gender

Female

Male

Age

18 to 24

25 to 49

50 to 64

Race/ethnicity

White

African American

Native American

Asian

Pacific islander

Hispanic

Other

Family income

$\$ 0$ to $\$ 24,999$

$\$ 25,000$ to $\$ 49,999$

$--$

$-$

$-$

--

$-$

--

$--$

1

0.996

Reference

[0.917, 1.082]

Reference

$[0.670,0.836]$

[0.597, 0.753]

Reference

[1.178, 1.570]

[0.757, 1.594]

[0.512, 1.030]

[1.197, 8.841]

[0.889, 1.269]

[0.866, 1.618]

0.906

[0.808, 1.016]

[0.798, 1.002]

184

0.894
Reference

[0.805, 1.082]

Reference

[0.697, 0.954]

Reference

[0.744, 0.950]

Reference

[1.387, 1.764]

Reference

[1.418, 1.864]

Reference

[0.870, 1.067]

Reference

$0.771^{* * *}$

$[0.670,0.889]$

[0.587, 0.783]

Reference

[1.388, 1.974]

[0.717, 1.719]

[0.504, 1.218]

[0.902, 14.49]

$[0.953,1.493]$

[0.831, 1.772]

1.193

1.213

[0.878, 1.157]

1.008

[0.793, 1.058] 


\begin{tabular}{|c|c|c|c|c|}
\hline \multicolumn{5}{|l|}{ Continued } \\
\hline$\$ 50,000$ to $\$ 74,999$ & $0.853^{*}$ & {$[0.741,0.983]$} & 0.857 & {$[0.724,1.014]$} \\
\hline$\$ 75,000$ to $\$ 99,999$ & 0.841 & {$[0.700,1.011]$} & 0.879 & {$[0.705,1.097]$} \\
\hline$\$ 100,000+$ & 1 & Reference & 1 & Reference \\
\hline \multicolumn{5}{|l|}{ Highest level of educational attainment } \\
\hline$>12$ th & 1 & Reference & 1 & Reference \\
\hline 12th or equivalent & 1.133 & {$[0.998,1.286]$} & 1.162 & {$[0.999,1.353]$} \\
\hline Associate degree & $1.335^{* * *}$ & {$[1.124,1.585]$} & $1.305^{*}$ & {$[1.061,1.606]$} \\
\hline Bachelor's degree & $1.202^{*}$ & {$[1.007,1.436]$} & $1.345^{* *}$ & {$[1.086,1.665]$} \\
\hline At least some graduate school & $1.310^{*}$ & {$[1.006,1.705]$} & $1.578^{* *}$ & {$[1.146,2.172]$} \\
\hline \multicolumn{5}{|l|}{ Marital status } \\
\hline Never married & 1 & Reference & 1 & Reference \\
\hline Married & 1.026 & {$[0.916,1.148]$} & 1.042 & {$[0.905,1.201]$} \\
\hline Widowed & 0.938 & {$[0.734,1.198]$} & 1.020 & {$[0.764,1.360]$} \\
\hline Divorced & 0.992 & {$[0.871,1.130]$} & 1.019 & {$[0.869,1.196]$} \\
\hline Separated & 0.973 & {$[0.791,1.197]$} & 1.071 & {$[0.831,1.381]$} \\
\hline \multicolumn{5}{|l|}{ Employment status } \\
\hline Unemployed & 1 & Reference & 1 & Reference \\
\hline Employed & $0.831^{*}$ & {$[0.720,0.958]$} & 0.839 & {$[0.702,1.003]$} \\
\hline Not in labor force & $0.818^{*}$ & {$[0.701,0.954]$} & 0.831 & {$[0.688,1.004]$} \\
\hline \multicolumn{5}{|l|}{ Geographic region } \\
\hline West & 1 & Reference & 1 & Reference \\
\hline Northeast & 0.972 & {$[0.841,1.122]$} & 0.927 & {$[0.777,1.105]$} \\
\hline Midwest & 1.000 & {$[0.880,1.138]$} & 0.954 & {$[0.815,1.117]$} \\
\hline South & $0.879^{*}$ & {$[0.777,0.995]$} & $0.857^{*}$ & {$[0.735,1.000]$} \\
\hline
\end{tabular}

Notes: (a) Adj. OR = adjusted odds ratio; $\mathrm{CI}=95 \%$ confidence interval. (b) ${ }^{*} p<0.05,{ }^{* *} p<0.01,{ }^{* * *} p<0.001$. (c) All estimates weighted. (d) $\mathrm{n}=$ 11,726 and $\mathrm{n}=8222$ for regressions (1) and (2), respectively.

long-term cessation trajectories. Under this assumption, an individual may be less likely to quit for a single day; nonetheless, she may be slowly reducing her tobacco consumption over the long run.

While 'any quit attempts' is a crucial indicator, other, equally important cessation measures must be explored within this paradigm. Future research might also investigate the prevalence of three and six-month cessation measures as well as the intention to quit among current smokers. The impact of physician advice may be distinct across cessation outcomes, influencing single day attempts but not having a durational effect on long-term cessation or shaping an individual's intention to quit.

In addition to alternative cessation measures, one might also explore the influence of varying clinical providers. An individual encounters several clinical providers, not just medical doctors-nurses, nurse practitioners, physician assistants, dentists, dental hygienists, etc. While advice from a medical doctor appears to significantly and positively impact the probability of any quit attempts, advice from other clinical providers potentially plays an important role as well. For instance, the literature suggests that nurse practitioners provide comparable primary care services (e.g., Mundinger et al., 2000); moreover, a growing number of patients see a nurse practitioner as their primary care provider. As such, nurse practitioners likely impact cessation efforts. Along these lines, the influence of clinician advice on cessation must be explored through the lens of non-physician providers if we are to capture a fuller picture. The 2010-2011 TUS-CPS contains data on cessation advice from a dentist, asking "During the PAST 12 MONTHS, did any dentist ADVISE you to stop smoking?” The TUS-CPS also includes the associated data relating to cessation strategies, offering a logical next step in this research. 
Machiavelli famously advised, “The end justifies the means.” Based on the current paper, one might instead argue that the "the means impacts the end." Given the steep knowledge gradient between patient and provider, physicians play an essential role in the clinical setting, acting as both a health leader and a health facilitator; however, this asymmetric information implies that clinical providers face an acute pressure not only to advise but to advise correctly. The findings emphasize that a physician can help initiate smoking cessation by simply broaching the topic of quitting with a patient; nevertheless, "the means impacts the end," i.e., the cessation strategy advised may further mediate the probability of successfully quitting or at least attempting to quit.

\section{References}

Centers for Disease Control and Prevention (CDC) (2013). Tobacco Use Goals and Key Areas. Smoking \& Tobacco Use. http://www.cdc.gov/tobacco/basic_information/healthy_people/goals/index.htminformation/healthy_people/goals/index.htm

Centers for Disease Control and Prevention (CDC) (2014). Current Cigarette Smoking Among Adults-United States, 2005-2012. Morbidity and Mortality Weekly Report (MMWR), 60, 29-34.

Chang, F. C., Sung, H. Y., Zhu, S. H., \& Chiou, S. T. (2014). Impact of the 2009 Taiwan Tobacco Hazards Prevention Act on Smoking Cessation. Addiction, 109, 140-146. http://dx.doi.org/10.1111/add.12344

Cohen, S. S., Sonderman, J. S., Mumma, M. T., Signorello, L. B., \& Blot, W. J. (2011). Individual and Neighborhood-Level Socioeconomic Characteristics in Relation to Smoking Prevalence among Black and White Adults in the Southeastern United States: A Cross-Sectional Study. BMC Public Health, 11, 877. http://dx.doi.org/10.1186/1471-2458-11-877

Fiore, M. C., Novotny, T. E., Pierce, J. P., Giovino, G. A., Hatziandreu, E. J., Newcomb, P. A., \& Davis, R. M. (1990). Methods Used to Quit Smoking in the United States: Do Cessation Programs Help? Jama, 263, 2760-2765. http://dx.doi.org/10.1001/jama.1990.03440200064024

Food and Drug Administration (FDA) (2013). Overview of the Family Smoking Prevention and Tobacco Control Act: Consumer Fact Sheet. http://www.fda.gov/tobaccoproducts/guidancecomplianceregulatoryinformation/ucm246129.htm

Gilpin, E. A., \& Pierce, J. P. (2002). Demographic Differences in Patterns in the Incidence of Smoking Cessation: United States 1950-1990. Annals of Epidemiology, 12, 141-150. http://dx.doi.org/10.1016/S1047-2797(01)00266-6

Keeler, C. (2013). Liberation Health and the Role of the Public Health Leader. Open Journal of Leadership, $2,82$. http://dx.doi.org/10.4236/ojl.2013.24012

Keeler, T. E., Marciniak, M., \& Hu, T. W. (1999). Rational Addiction and Smoking Cessation: An Empirical Study. The Journal of Socio-Economics, 28, 633-643. http://dx.doi.org/10.1016/S1053-5357(99)00038-4

Jemal, A., Thun, M., Xue, Q. Y., Hartman, A. M., Cokkinides, V., Center, M. M., \& Ward, E. M. (2011). Changes in Smoking Prevalence among US Adults by State and Region: Estimates from the Tobacco Use Supplement to the Current Population Survey, 1992-2007. BMC Public Health, 11, 512. http://dx.doi.org/10.1186/1471-2458-11-512

Mundinger, M. O., Kane, R. L., Lenz, E. R., Totten, A. M., Tsai, W. Y., Cleary, P. D., \& Shelanski, M. L. (2000). Primary Care Outcomes in Patients Treated by Nurse Practitioners or Physicians: A Randomized Trial. Jama, 283, 59-68. http://dx.doi.org/10.1001/jama.283.1.59

Parrott, S., \& Godfrey, C. (2004). Economics of Smoking Cessation. Bmj, 328, 947-949. http://dx.doi.org/10.1136/bmj.328.7445.947

Phelps, C. (2012). Health Economics (5th ed.). Upper Saddle River, NJ: Pearson Education.

Pierce, J. P., White, M. M., \& Messer, K. (2009). Changing Age-Specific Patterns of Cigarette Consumption in the United States, 1992-2002: Association with Smoke-Free Homes and State-Level Tobacco Control Activity. Nicotine \& Tobacco Research, 11, 171-177. http://dx.doi.org/10.1093/ntr/ntp014

Rathert, C., Huddleston, N., \& Pak, Y. (2011). Acute Care Patients Discuss the Patient Role in Patient Safety. Health Care Management Review, 36, 134-144. http://dx.doi.org/10.1097/HMR.0b013e318208cd31

Townsend, J., Roderick, P., \& Cooper, J. (1994). Cigarette Smoking by Socioeconomic Group, Sex, and Age: Effects of Price, Income, and Health Publicity. Bmj, 309, 923-927. http://dx.doi.org/10.1136/bmj.309.6959.923

Trinidad, D. R., Pérez-Stable, E. J., Messer, K., White, M. M., \& Pierce, J. P. (2010). Menthol Cigarettes and Smoking Cessation among Racial/Ethnic Groups in the United States. Addiction, 105, 84-94. http://dx.doi.org/10.1111/j.1360-0443.2010.03187.x

US Department of Health and Human Services (2012). About Healthy People. Healthy People 2020. http://www.healthypeople.gov/2020/about/

US Department of Health and Human Services (HHS) (2014) The Health Consequences of Smoking: 50 Years of Progress. A Report of the Surgeon General. Atlanta, GA: US Department of Health and Human Services, Centers for Disease Control and Prevention, National Center for Chronic Disease Prevention and Health Promotion, Office on Smoking and Health. 